Published in final edited form as:

J Cancer Educ. 2019 June ; 34(3): 614-622. doi:10.1007/s13187-018-1374-0.

\title{
Survivorship care plan implementation in US cancer programs: A national survey of cancer care providers
}

\author{
Sarah A. Birken, PhD, \\ Department of Health Policy and Management, Gillings School of Global Public Health, The \\ University of North Carolina at Chapel Hill, 1103E McGavran-Greenberg, 135 Dauer Drive, \\ Campus Box 7411, Chapel Hill, NC 27599-7411 \\ Sarah Raskin, PhD, MPH, \\ L. Douglas Wilder School of Government and Public Affairs, Virginia Commonwealth University

\section{Yuqing Zhang,} \\ Institute for Patient-Centered Initiatives \& Health Equity, The George Washington University \\ Cancer Center

\section{Gema Lane,} \\ Institute for Patient-Centered Initiatives \& Health Equity, The George Washington University \\ Cancer Center
}

\author{
Alexandra Zizzi, MSPH, and \\ Department of Health Policy and Management, Gillings School of Global Public Health, The \\ University of North Carolina at Chapel Hill

\section{Mandi Pratt-Chapman, MA} \\ Institute for Patient-Centered Initiatives \& Health Equity, The George Washington University \\ Cancer Center
}

\section{INTRODUCTION}

There are more than 15.5 million cancer survivors in the United States (US) [1]. Cancer and its treatment may have late and long-term physical, psychosocial, financial, sexual and spiritual effects on survivors [2]. These effects can be addressed through coordinated care from follow-up care providers (e.g., primary care providers [PCPs], oncologists); however, coordination of survivors' care is often poor, at times resulting in the duplication or omission of recommended services [3-7], poor health outcomes [6, 8-11], and significant costs to survivors and the US healthcare system [12]. To improve coordination of care and outcomes for survivors, organizations including the American College of Surgeons' Commission on Cancer and National Accreditation Program of Breast Centers require cancer care providers to develop and deliver survivorship care plans (SCPs) to survivors and their follow-up care providers [12-16]. SCPs are written documents that ideally include treatment summary

Corresponding author: Phone: (919) 445-0774, Fax: (919) 843-6308 birken@unc.edu (Sarah A. Birken, PhD). 
information (e.g., diagnosis, stage, treatments), plans for follow-up care (e.g., surveillance, preventive services), and recommended division of responsibilities among follow-up care providers.

Although SCP use recommendations and requirements are widespread, the effectiveness of SCPs remains unclear. Qualitative and observational studies have found that survivors and PCPs benefit from SCPs [17]; however, to date, 7 papers from 8 randomized controlled trials (RCTs) of SCPs' effectiveness have reported largely null results [18-24]. Null results may be due, in part, to variation in SCP implementation. SCP implementation in US cancer programs remains at an estimated 12-43\% [25-29]. Salz et al. (2012) found that $43 \%$ of National Cancer Institute (NCI)-designated cancer programs used SCPs for breast cancer survivors [28]. In their study of Massachusetts cancer care providers, Merport et al. (2012) found that just $14 \%$ developed SCPs [29]. Less than $10 \%$ of the nationally-representative sample of medical oncologists in Forsythe et al.'s 2013 study reported always or almost always developing SCPs for survivors [30].

Variation in implementation likely influences SCPs' effectiveness; if SCPs are not successfully implemented, they are unlikely to produce desired results. Researchers have identified barriers to SCP implementation including insufficient resources of time, staff, training, templates and funding [26,28,30,31], yet few studies have articulated the processes used to implement SCPs. As such, our study objective was to characterize the processes used to implement SCPs in practice. Understanding how cancer care providers identify survivors who are eligible for SCPs, refer them for SCPs, develop SCPs, and deliver SCPs to survivors and their follow-up care providers illuminates the challenges to implementing SCPs, opportunities for improving SCP implementation processes, and promising approaches to implementing SCPs.

\section{MATERIALS AND METHODS}

\section{Survey development}

In response to several questions regarding SCPs posted on the Association of Community Cancer Center's (ACCC) membership-only web-based ACCC Exchange, the PI (MPC) fielded a brief survey to clarify providers' informational needs regarding SCPs. Twenty-nine individuals responded and were invited to participate in a Survivorship Special Interest Group (S-SIG), a group created as part of the Patient-Centered Outcomes Research Institutefunded Generation And Translation of Evidence (GATE) Cancer Community of Practice contract. S-SIG members participated in conference calls to develop a survey to identify informational gaps in providers' SCP-related needs. The PI (MPC) drafted the first iteration of the survey based on feedback from the S-SIG. After review by the S-SIG to confirm that the survey reflected their feedback, the co-PI (SB) revised the draft with an eye to the stages of SCP implementation: 1) identify survivors who are eligible to receive SCPs; 2) refer survivors to receive SCPs; 3) develop, 4) save and/or 5) update SCPs; and/or 6) deliver SCPs to survivors or other providers. We then conducted cognitive interviews with three providers who use SCPs to improve content validity of individual questions, and edited questions based on feedback. The final survey consisted of six sections representing each stage of SCP implementation, each with approximately 10 items, and a demographic section regarding 
characteristics of respondents' cancer programs. In most cases, items allowed respondents to select multiple response options.

\section{Study Participants}

We recruited a convenience sample of US health care providers who are responsible for one or more of the stages of SCP implementation. In particular, we were interested in providers' perspectives on the processes used to execute each of these stages (e.g., person responsible, prompt to initiate). George Washington University employees were not eligible to participate due to institutional research restrictions. We recruited respondents by sending customized emails to the following listservs: the Association of Community Cancer Centers (approximately 23,000 members), the Academy of Oncology Nurse \& Patient Navigators (approximately 5,000 members), the Commission on Cancer (representatives from approximately 1,500 cancer programs), and the Oncology Nursing Society (approximately 39,000 members). In addition, five states funded by the Centers for Disease Control and Prevention to advance survivorship in their states were invited to participate (Indiana, Louisiana, Michigan, South Dakota, and Washington State). The study team also invited the approximately 35 members of the S-SIG and other stakeholders within our networks (approximately 10) to share the survey invitation with their contacts. Collectively, these groups represented nearly 69,000 potential participants who may have received our emails. To optimize recruitment, we used Dillman's method of repeated contact [32], adapted based on organizational policies of partners regarding number of times their membership could be contacted.

\section{Data Collection}

We fielded the one-time, anonymous online survey resulting from the S-SIG's efforts between November 2016 and January 2017. We administered the survey and managed study data using REDCap (Research Electronic Data Capture), a secure, web-based survey software. We programmed skip logics into the survey to direct respondents to the stages of SCP implementation in which they engaged. Upon completing the survey, respondents were invited to submit their contact information, using a separate Survey Monkey link, to be entered into a draw for a $\$ 50$ gift card. The Institutional Review Board at the George Washington University exempted the study from human subjects review.

\section{Statistical Analysis}

To characterize SCP implementation in practice, we report descriptive statistics including percentages, medians, and ranges. We report medians and ranges because of substantial variation and right-skewing in the data. Out of the interested potential participants, we only included those who met the eligibility criteria.

\section{RESULTS}

\section{Respondent characteristics}

Of the approximately 69,000 potential participants who may have received our recruitment emails, the study sample consisted of 395 survey respondents from 47 states and Washington, DC, for an approximate response rate of $0.6 \%$. Respondents primarily included 
nurse navigators (44\%), registered nurses (30\%), and oncology nurse practitioners (10\%) (Table 1). Respondents had multiple roles in SCP use. Eighty percent of respondents were responsible for more than one stage of SCP implementation, and nearly $51 \%$ were responsible for five or more stages.

Respondents reported that the median annual incidence of cancer in their programs was $\mathrm{n}=700$ patients (range $=1-8000$ ). Forty-six percent $(46 \%)$ of respondents worked in community hospital comprehensive cancer centers, and $27 \%$ worked in community hospital cancer programs (Table 1). Respondents' cancer programs were members of professional organizations including the American College of Surgeon's Commission on Cancer (81\%), the Association of Community Cancer Centers (38\%), and the American Society for Clinical Oncology's (ASCO) Quality Oncology Practice Initiative (36\%).

\section{SCP implementation processes}

Table 2 describes the processes (rows) used to execute each stage of SCP implementation (columns). For example, the first row describes the prompt to initiate (process) each stage of SCP implementation (e.g., identifying survivors, delivering SCPs). Immediately below, we describe cross-cutting issues that apply to multiple stages of SCP implementation. Following that section, we examine key issues pertaining to selected stages of SCP implementation.

\section{Cross-cutting issues}

SCPs were primarily used for three cancer survivor groups: The most common survivor groups for which SCPs were used included breast (85\%), colon or rectal (59\%), and lung $(53 \%)$. More than $30 \%$ of respondents reported that SCPs were used for prostate, head and neck, endometrial, ovarian, and lymphoma survivors. Respondents reported that SCPs were seldom used for brain, myeloma, or leukemia survivors (Table 1).

Timing of SCP implementation varied across US cancer programs: Sixty-one percent $(61 \%)$ of respondents reported that SCPs were developed within three months of completion of adjuvant therapy; $40 \%$ reported developing SCPs more than three months after completion of primary treatment or adjuvant therapy. Likewise, $65 \%$ of respondents reported that SCPs were delivered to survivors within three months of completion of adjuvant therapy, but another 50\% reported doing so more than three months after treatment or therapy are completed. That many respondents selected multiple response options suggests that the timing of SCP implementation varies within, as well as across cancer programs.

SCP implementation primarily fell to nurse navigators: Approximately half of respondents indicated that nurse navigators are responsible for each stage of SCP implementation in their cancer programs. Many respondents indicated that oncology nurse practitioners or oncologists develop the follow-up care portion of SCPs (22\% and 26\%, respectively) and deliver SCPs to survivors (31\% and 24\%, respectively). Cancer registry staff, oncology physician assistants, and social workers were seldom identified as responsible for SCP implementation. 
Providers used suboptimal methods of implementing SCPs: Processes that could have been automated because they did not require discussion of patient issues often occurred inperson or via phone. Few SCPs were developed in electronic health records (EHRs; 43\%); of the respondents who reported developing SCPs in EHRs, just 46\% indicated that at least a portion of original or updated information in SCPs was autopopulated (Table 2). In contrast, processes that may have benefitted from direct contact occurred indirectly. Although more than half of respondents reported delivering SCPs to survivors in person, nearly a third reported doing so via postal service. More than half of respondents (58\%) reported that SCPs (original and updated) were delivered to follow-up care providers via fax.

Implementing SCPs was time-consuming: On average, respondents reported spending more than two hours per SCP to complete all stages of implementation, even among those who automated some stages of SCP implementation (Table 2). Respondents reported that the most time-consuming stages were developing the treatment summary (median $=30$ minutes; range $=0.3-240$ minutes) and delivering the SCP to survivors (median $=39$ minutes; range $=$ 0-240 minutes). The least time-consuming stage was delivering SCPs to follow-up care providers (median $=10$ minutes; range $=0-280$ minutes).

Financial commitment to SCP use was lacking: Most respondents (97-99\%) reported receiving no external funding to support SCP implementation. Eighteen percent $(18 \%)$ of respondents were reimbursed from private or public payers for SCP delivery to survivors (Table 2). Notably, of the approximately 140 minutes that it took respondents to see SCPs through all stages, respondents reported that only the delivery of SCPs to survivors, which took less than one-third of the total time required to complete SCP implementation, was eligible for reimbursement.

\section{Selected stages of SCP implementation}

Stage 1: Template creation and revision: Most commonly, SCP templates were developed within cancer programs (42\%). Respondents reported that follow-up care plan content was most often informed by National Comprehensive Cancer Network (NCCN) (80\%) and ASCO (60\%) guidelines. A plurality of respondents (39\%) reported that SCP templates in their cancer program were revised once a year and that more than one colleague reviewed template revisions (63\%). Most respondents reported that revisions to SCP templates were prompted by changes in guidelines or recommendations $(63 \%)$ or changes in stakeholders' (e.g., survivors, caregivers) needs (59\%; Table 3).

Stage 2: Identifying survivors who are eligible for SCPs: Forty-four percent (44\%) of respondents indicated that they lacked a system for identifying survivors as eligible for SCPs; $73 \%$ reported doing so on a case-by-case basis. Most respondents reported that, subsequent to identification, an SCP would be developed (82\%) and delivered to a survivor during a clinical visit (74\%; Table 2).

Stage 3: Referring survivors for SCPs: Seventy percent (70\%) of respondents reported that referral was for SCP delivery during a patient visit; and 20\% reported that referral was for SCP delivery via postal service, fax, email, or patient portal. There was substantial 
variation in the provider to whom survivors were referred. Most frequently, survivors were referred to nurse practitioners (NPs) (40\%), nurse navigators (40\%), oncologists (35\%), and/or registered nurses (RNs) (28\%). That respondents selected multiple options suggests that to whom survivors were referred for SCPs varied within, as well as across cancer programs.

Stage 5: Delivering SCPs to survivors: Most respondents reported that SCPs were delivered during a visit not specific to survivorship (65\%) or a survivorship-specific visit $(55 \%)$ in the same location as cancer treatment was provided (52\%) or in a separate room affiliated with the cancer program (52\%). Thirty-two percent of respondents reported that SCPs were delivered via postal service. Most respondents reported that survivors received other informational materials along with SCPs (85\%). Twenty-four respondents (24\%) reported also providing consultation when delivering SCPs (Table 2).

Stage 7: Updating SCPs: Respondents reported relying most often on NCCN or ASCO guidelines to update original SCP content. Most respondents reported delivering updated SCPs to survivors (75\%) and follow-up care providers (51\%) and reported that the process for delivery did not differ from the process for delivering the original SCP (86\%; Table 2).

\section{DISCUSSION}

We sought to characterize SCP implementation in US cancer programs, a topic that has received little attention in extant studies. In so doing, we identified several persistent challenges in implementing SCPs. First, we found that SCP implementation primarily fell to nurse navigators. To the extent that this represents one or a small number of individuals bearing the burden, SCP implementation is unlikely to be consistent; if the few people charged with the task are unavailable, SCPs will not be implemented. Indeed, in another of our studies, we found that tasking appropriate employees with SCP implementation and ensuring that SCP implementation does not fall on individual employees was critical for successful SCP implementation [(Authors' names redacted for blinding purposes):

Determinants of successful survivorship care plan implementation: results from a qualitative study. Manuscript under review].

Second, we found that providers used suboptimal methods of implementing SCPs. When warm handoffs were warranted to convey important patient information (e.g., discussing nuances of a survivor's condition with a PCP), processes were automated, and when automation might have expedited processes (e.g., developing SCPs), providers communicated in-person or via phone. These suboptimal modes of communication are likely to reduce efficiency and quality by, for example, decreasing the information transmitted regarding the nuances of a survivor's case and increasing the time required to develop SCPs. Solet et al. (2005) recommended that patient handoffs in the hospital setting occur in person in order to adequately communicate the full range of patient care needs to the receiving physician. The authors found that commonly-used indirect measures (e.g. medical chart) left ambiguities in the care process for which the receiving physician could not easily find answers, and could lead to errors in care [33]. 
Third, SCP implementation remains time-consuming. This finding is consistent with extant studies suggesting that developing SCPs can take up to 1.5 hours [34] and expands on those studies by identifying (1) the scope of the issue based on responses from hundreds of US cancer programs and (2) additional bottlenecks in SCP implementation, including delivering the SCP to survivors. Identifying methods of streamlining these processes without compromising quality will be critical for facilitating implementation and promoting SCPs' effectiveness, particularly in light of evidence that suggests that clinicians are willing to allocate 20 minutes or less to SCP development [30].

Fourth, we found that SCP implementation is underfunded, potentially influencing the proficiency and consistency with which SCPs are implemented as cancer programs struggle to comply with requirements while providing billable services. Reimbursement for SCP implementation is uncommon overall. Even among practices that successfully obtain reimbursement for SCPs, few processes - and so, only a partial percentage of effort - are compensated. To date, a Medicare code exists for providers to document SCP development, but funding is not currently available to reimburse providers for these services. Medicare and other third-party payment may be necessary to promote more widespread SCP implementation.

Fifth, we found substantial variation in SCP implementation both across and within cancer programs, suggesting disparities in survivorship care. This is consistent with Salz et al.'s (2012) finding of variation in SCP content and subsequent lack of adherence to recommended survivorship care guidelines [28]. To the extent that variation contributes to disparities in survivorship care, promoting more consistent SCP implementation may limit existing disparities in survivorship care.

Several limitations of this study should be noted. With a convenience sample, we reached a small fraction of the nearly 69,000 potential participants who may have received our recruitment email. Therefore, we are unable to generalize to all US cancer programs; however, our study was intended to be exploratory. Further, respondents came from 14 types of cancer programs in 47 states and Washington, DC, suggesting excellent representation. Another limitation is that we did not validate or assess survey items' reliability, although we conducted three cognitive interviews to promote survey item quality. Social desirability bias may have caused respondents to over-report the quality of SCP implementation. Further, providers who agreed to participate in the study may have been more likely to respond if their programs successfully implemented SCPs; however, the variation in study outcomes suggests otherwise.

Despite these limitations, our study represents the largest survey to date of SCP implementation of which we are aware and reflects the engagement of diverse clinical stakeholders seeking to improve practice through understanding of facilitators and barriers. Qualitative work is needed to understand SCP implementation, with specific attention to contextual factors that can facilitate, inhibit, and otherwise shape implementation, including consistency and variation in implementation. Future studies should also identify models of SCP implementation emerging from our survey data, allowing us to assess relationships between these models and desired outcomes of SCP use; if SCP implementation influences 
effectiveness, extant RCTs may be inconclusive due to poor implementation, not SCPs' inherent (in)effectiveness.

\section{Acknowledgments}

This project was an outgrowth of engagement activities supported by a Patient-Centered Outcomes Research Institute (PCORI) Eugene Washington PCORI Engagement Award (1426-GWU). This publication was supported by Award Number UL1TR001876 from the NIH National Center for Advancing Translational Sciences. Infrastructure that made this project possible was partially supported by Award Number UL1TR001876 from the NIH National Center for Advancing Translational Sciences. SB also received funding from the National Institutes of Health through Grant KL2TR001109. The contents of this publication are solely the responsibility of the authors and do not necessarily represent the views of the Patient-Centered Outcomes Research Institute (PCORI), its Board of Governors, or Methodology Committee, or the official views of the National Center for Advancing Translational Sciences or the National Institutes of Health. We appreciate the input of the Survivorship Special Interest Group, George Washington Cancer Center's PCORI-funded Generation and Translation of Evidence: An Oncology Community of Practice, a group created as a result of PCORI Contract 1426-GWU, as well as collaborating organizations who contributed to sample recruitment.

\section{References}

1. Ganz, Patricia A. 2001; Late effects of cancer and its treatment. Semin Oncol Nurse. 17(4):241-248.

2. Hewitt, Maria; Greenfield, Sheldon; Stovall, Ellen, editors. From Cancer Patient to Cancer Survivor: Lost in Transition. Washington, DC: The National Academies Press; 2005.

3. Urquhart, Robin; Folkes, Amy; Porter, Geoffrey; , et al. 2012; Population-based longitudinal study of follow-up care for colorectal cancer patients in Nova Scotia. J Oncol Pract. 8(4):246-252. [PubMed: 23180991]

4. Grunfeld, Eva; Hodgson, David C; Del Guidice, M Elisabeth; , et al. 2010; Population-based longitudinal study of follow-up care for breast cancer survivors. J Oncol Pract. 6(4):174-181. [PubMed: 21037867]

5. Hahn, Erin E; Hays, Ron D; Kahn, Katherine L; , et al. 2013; Use of imaging and biomarker tests for post-treatment care of early stage breast cancer survivors. Cancer. 119(24):4316-4326. [PubMed: 24105101]

6. Hahn, Erin E; Tang, Tania; Lee, Janet S; , et al. 2016; Use of post-treatment imaging and biomarkers in early stake breast cancer survivors: inappropriate surveillance or necessary care? Cancer. 122(6): 908-916. [PubMed: 26650715]

7. Cheung, Winson Y; Neville, Bridget A; Cameron, Danielle B; , et al. 2009; Comparisons of patient and physician expectations for cancer survivorship care. J Clin Oncol. 27(15):2489-2495. [PubMed: 19332716]

8. Mallinger, Julie B; Griggs, Jennifer J; Shields, Cleveland G. 2005; Patient-centered care and breast cancer survivors' satisfaction with information. Patient Educ Couns. 57(3):342-349. [PubMed: 15893218]

9. Nicolaije, Kim AH; Husson, Olga; Ezendam, Nicole PM; , et al. 2012; Endometrial cancer survivors are unsatisfied with received information about diagnosis, treatment and follow-up: a study from the population-based PROFILES registry. Patient Educ Couns. 88(3):427-435. [PubMed: 22658248]

10. Siegel, Rebecca; DeSantis, Carol; Virgo, Katherine; , et al. 2012; Cancer treatment and survivorship statistics, 2012. CA Cancer J Clin. 62(4):220-241. [PubMed: 22700443]

11. Levit, Laura A, , et al., editors. Institute of Medicine. Delivering High-Quality Cancer Care: Charting a New Course for a System in Crisis. Washington, DC: National Academy of Sciences; 2013.

12. American College of Surgeons National Accreditation Program for Breast Cancers. Clarifications/ Changes to NAPBC Standards. https://www.facs.org/quality-programs/napbc/standards/changes. Accessed 12 October 2017

13. National Cancer Survivorship Resource Center. Systems Policy and Practice: Clinical Survivorship Care. Atlanta, GA: American Cancer Society; 2011. https://www.cancer.org/content/dam/cancer- 
org/cancer-control/en/reports/systems-policy-and-practice-clinical-survivorship.pdf. Accessed 12 October 2017

14. McCabe, Mary S; Bhatia, Smita; Oeffinger, Kevin C; , et al. 2013; American Society of Clinical Oncology statement: achieving high-quality cancer survivorship care. J Clin Oncol. 31(5):631640. [PubMed: 23295805]

15. Commission on Cancer, American Cancer Society. Cancer Program Standards 2012: Ensuring Patient-Centered care. Atlanta, GA: American College of Surgeons; 2012. https://www.facs.org/ / media/files/quality\%20programs/cancer/coc/programstandards2012.ashx. Accessed 12 October 2017

16. Shalom, Marina M; Hah, Erin E; Casillas, Jacqueline; , et al. 2011; Do survivorship care plans make a difference? A primary care provider perspective. J Oncol Pract. 7(5):314-318. [PubMed: 22211129]

17. Grunfeld, Eva; Julian, Jim A; Pond, Gregory; , et al. 2011; Evaluating survivorship care plans: results of a randomized, clinical trial of patients with breast cancer. J Clin Oncol. 29(36):47554762. [PubMed: 22042959]

18. Boekhout, Annelies H; Maunsell, Elizabeth; Pond, Gregory R; , et al. 2015; A survivorship care plan for breast cancer survivors: extended results of a randomized clinical trial. J Cancer Surviv. 9:683-691. [PubMed: 25896265]

19. Coyle, Doug; Grunfeld, Eva; Coyle, Kathryn; , et al. 2013; Cost effectiveness of a survivorship care plan for breast cancer survivors. J Oncol Pract. 10(2):e86-e92. [PubMed: 24326740]

20. Brothers, Brittany M; Easley, Andrea; Salani, Ritu; , et al. 2013; Do survivorship care plans impact patients' evaluations of care? A randomized evaluation with gynecologic oncology patients. Gynecol Oncol. 129(3):554-558. [PubMed: 23474344]

21. Hershman, Dawn L; Greenlee, Heather; Awad, Danielle; , et al. 2013; Randomized controlled trial of a clinic-based survivorship intervention following adjuvant therapy in breast cancer survivors. Breast Cancer Res Treat. 138:795-806. [PubMed: 23542954]

22. Nicolaije, Kim AH; Ezendam, Nicole PM; Vos, M Caroline; , et al. 2015; Impact of an automatically generated cancer survivorship care plan on patient-reported outcomes in routine clinical practice: longitudinal outcomes of a pragmatic cluster randomized trial. J Clin Oncol. 33(31):3550-3559. [PubMed: 26304900]

23. de Rooij, Belle H; Ezendam, Nicole PM; Nicolaije, Kim AH; , et al. 2017; Effects of survivorship care plans on patient reported outcomes in ovarian cancer during 2-year follow-up - the ROGY care trial. Gynecol Oncol. 145(2):319-328. [PubMed: 28283195]

24. Birken, Sarah A; Deal, Allison M; Mayer, Deborah K; Weiner, Bryan J. 2014; Determinants of survivorship care plan use in United States programs. J Canc Educ. 29(4):720-727.

25. Birken, Sarah A; Deal, Allison M; Mayer, Deborah K; Weiner, Bryan J. 2014; Following through: the consistency of survivorship care plan use in United States cancer programs. J Cancer Educ. 29(4):689-697. [PubMed: 24577781]

26. Birken, Sarah A; Mayer, Deborah K; Weiner, Bryan J. 2013; Survivorship care plans: prevalence and barriers to use. J Canc Educ. 28(2):290-296.

27. Salz, Talya; Oeffinger, Kevin C; McCabe, Mary S; , et al. 2012; Survivorship care plans in research and practice. CA Cancer J Clin. 62:101-117. [PubMed: 22241452]

28. Merport, Anna; Lemon, Stephenie C; Nyambose, Joshua; Prout, Marianne N. 2012; The use of cancer treatment summaries and care plans among Massachusetts physicians. Support Care Cancer. 20:1579-1584. [PubMed: 22526150]

29. Forsythe, Laura P; Parry, Carla; Alfano, Catherine M; , et al. 2013; Gaps in survivorship care plan delivery and potential benefits to survivorship care [abstract]. J Clin Oncol. 31

30. Hewitt, Maria E; Bamundo, Annette; Day, Rebecca; Harvey, Catherine. 2007; Perspectives on posttreatment cancer care: qualitative research with survivors, nurses, and physicians. J Clin Oncol. 25:2270-2273. [PubMed: 17538172]

31. Campbell, Bruce H; Massey, Becky L; Myers, Katherine B. 2012; Survivorship care plans for patients with head and neck cancer. Arch Otolaryngol Head Neck Surg. 138:1116-1119. [PubMed: 23247230]

J Cancer Educ. Author manuscript; available in PMC 2020 June 01. 
32. Dillman, Don A. Mail and Telephone Surveys: The Total Design Method. New York, NY: John Wiley \& Sons; 1978.

33. Solet, Darrell J; Norvell, J Michael; Rutan, Gale H; Frankel, Richard M. 2005; Lost in translation: challenges and opportunities in physician-to-physician communication during patient handoffs. Acad Med. 80(12):1094-1099. [PubMed: 16306279]

34. Jefford, Michael; Lotfi-Jam, Kerryann; Baravelli, Carl; , et al. 2011; Development and pilot testing of a nurse-led posttreatment support package for bowel cancer survivors. Cancer Nurs. 34(3):E110 . 
Table 1

Characteristics of respondents, respondents' organizations, and survivorship care plan (SCP) implementation

\begin{tabular}{|c|c|}
\hline Characteristics & $\%$ \\
\hline \multicolumn{2}{|l|}{ Respondent's role in SCP use $(n=394)$} \\
\hline Identify survivors who are eligible to receive SCPs & 72.3 \\
\hline Develop SCPs & 66.0 \\
\hline Create and/or revise SCP templates & 62.7 \\
\hline Save SCPs & 53.6 \\
\hline Deliver SCPs to cancer survivors & 53.6 \\
\hline Deliver SCPs to survivors' follow-up care providers & 48.2 \\
\hline Update SCPs & 36.5 \\
\hline Refer survivors to receive SCPs & 28.4 \\
\hline None of the above & 0.0 \\
\hline \multicolumn{2}{|l|}{ Respondent's current position $(\mathrm{n}=394)$} \\
\hline Nurse Navigator & 44.2 \\
\hline Registered Nurse & 29.9 \\
\hline Oncology Nurse Practitioner & 9.6 \\
\hline Cancer registry staff & 3.8 \\
\hline Patient Navigator (not a nurse or social worker) & 3.3 \\
\hline Social Worker & 3.3 \\
\hline Nurse Practitioner (not oncology-based) & 2.8 \\
\hline Health Educator & 2.0 \\
\hline Oncologist (physician) & 1.5 \\
\hline Physician other than oncologist & 1.5 \\
\hline Oncology Physician Assistant & 1.3 \\
\hline Physician Assistant (not oncology-based) & 0.8 \\
\hline Medical Director & 0.0 \\
\hline Other & 22.1 \\
\hline Decline to respond & 1.3 \\
\hline Annual incident cancers [median (range)] $(\mathrm{n}=292)$ & $700(1-8000)$ \\
\hline \multicolumn{2}{|l|}{ Cancer program type $(n=292)$} \\
\hline Community hospital comprehensive & 45.5 \\
\hline Community hospital & 27.4 \\
\hline Affiliate & 12.7 \\
\hline Hospital associate & 11.3 \\
\hline Teaching hospital & 10.6 \\
\hline Freestanding & 7.9 \\
\hline National Cancer Institute-designated comprehensive & 5.5 \\
\hline National Cancer Institute-designated & 4.1 \\
\hline Integrated & 2.7 \\
\hline Network & 2.1 \\
\hline Pediatric & 1.7 \\
\hline
\end{tabular}

J Cancer Educ. Author manuscript; available in PMC 2020 June 01. 


\begin{tabular}{|c|c|}
\hline Characteristics & $\%$ \\
\hline National Cancer Institute-designated cancer network & 1.4 \\
\hline Veterans Affairs & 1.4 \\
\hline Pediatric cancer program component & 0.7 \\
\hline Don't know & 1.7 \\
\hline Decline to respond & 0.7 \\
\hline \multicolumn{2}{|l|}{ Organization Membership $(\mathrm{n}=292)$} \\
\hline American College of Surgeons Commission on Cancer & 80.8 \\
\hline Association of Community Cancer Centers & 38.4 \\
\hline American Society for Clinical Oncology Quality Oncology Practice Initiative & 36.3 \\
\hline National Comprehensive Cancer Network & 26.0 \\
\hline NCI Community Cancer Centers Program & 5.8 \\
\hline LIVESTRONG Foundation (formerly Lance Armstrong Foundation) & 3.8 \\
\hline National Coalition for Cancer Survivorship & 3.1 \\
\hline Don't know & 12.0 \\
\hline Decline to respond & 2.4 \\
\hline \multicolumn{2}{|l|}{ Tumor groups for which SCPs are used $(n=394)$} \\
\hline Breast & 85.0 \\
\hline Colon or rectal (including anal) & 58.6 \\
\hline Lung & 53.3 \\
\hline Prostate & 43.4 \\
\hline Head and neck & 42.1 \\
\hline Endometrial & 32.2 \\
\hline Ovarian & 31.2 \\
\hline Lymphoma (Hodgkin and non-Hodgkin) & 31.0 \\
\hline Testicular & 25.1 \\
\hline Kidney & 24.1 \\
\hline Melanoma & 23.9 \\
\hline Thyroid & 20.1 \\
\hline Brain & 18.5 \\
\hline Myeloma & 14.0 \\
\hline Leukemia & 12.2 \\
\hline Clinical trials patients & 6.6 \\
\hline Other & 7.6 \\
\hline Don't know & 1.0 \\
\hline Decline to respond & 1.3 \\
\hline \multicolumn{2}{|l|}{ Organizational system for identifying survivors $(\mathrm{n}=257)$} \\
\hline Non-automated system & 45.9 \\
\hline No system & 44.4 \\
\hline Automated system & 4.7 \\
\hline Other & 12.5 \\
\hline Decline to respond & 0.8 \\
\hline
\end{tabular}




\begin{tabular}{lc}
\hline Characteristics & $\%$ \\
\hline On a case-by-case basis & 73.2 \\
Patient receives care in a particular tumor group & 23.0 \\
Other & 21.0 \\
Don't know & 0.4 \\
Decline to respond & 0.8 \\
\hline
\end{tabular}




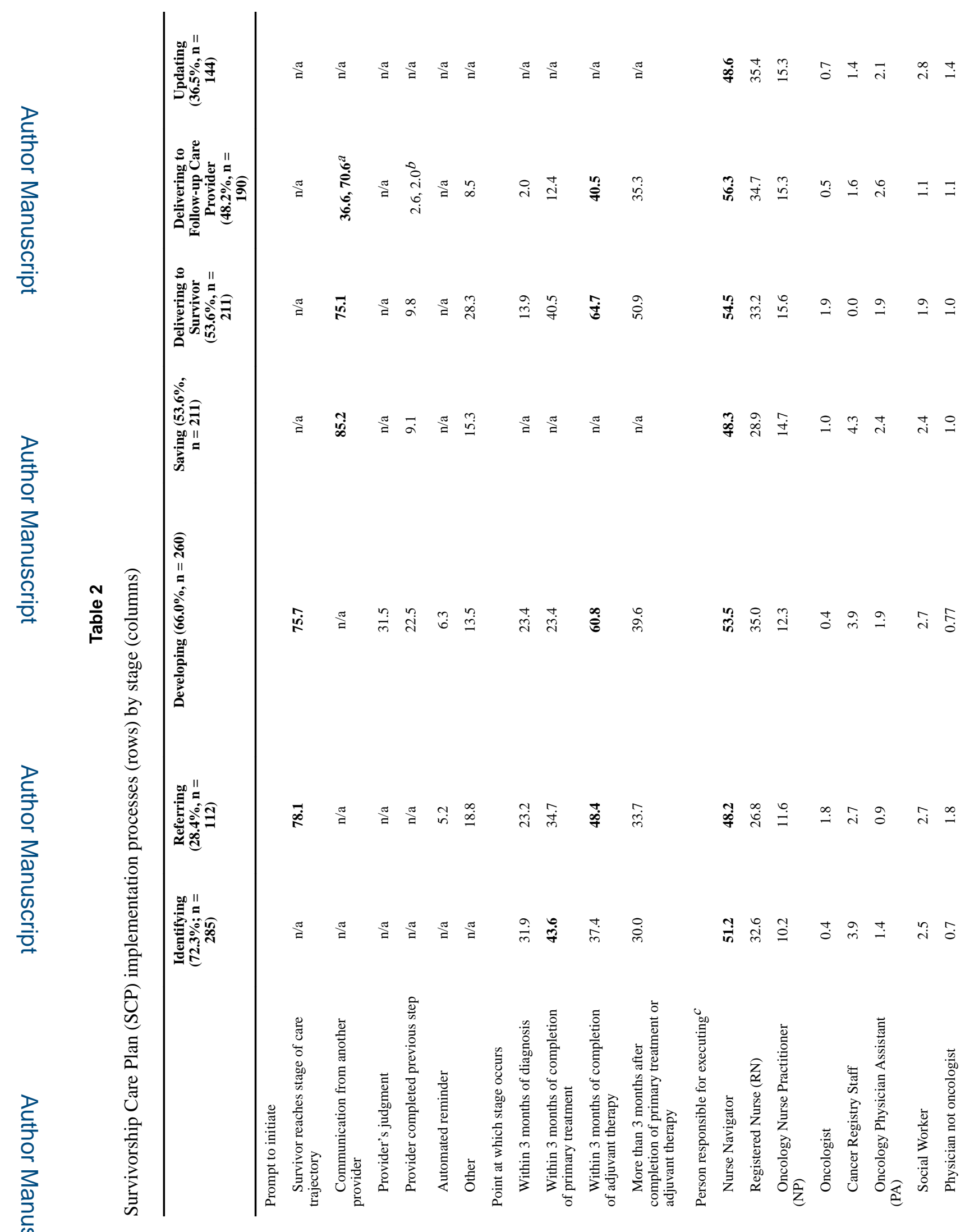

J Cancer Educ. Author manuscript; available in PMC 2020 June 01. 


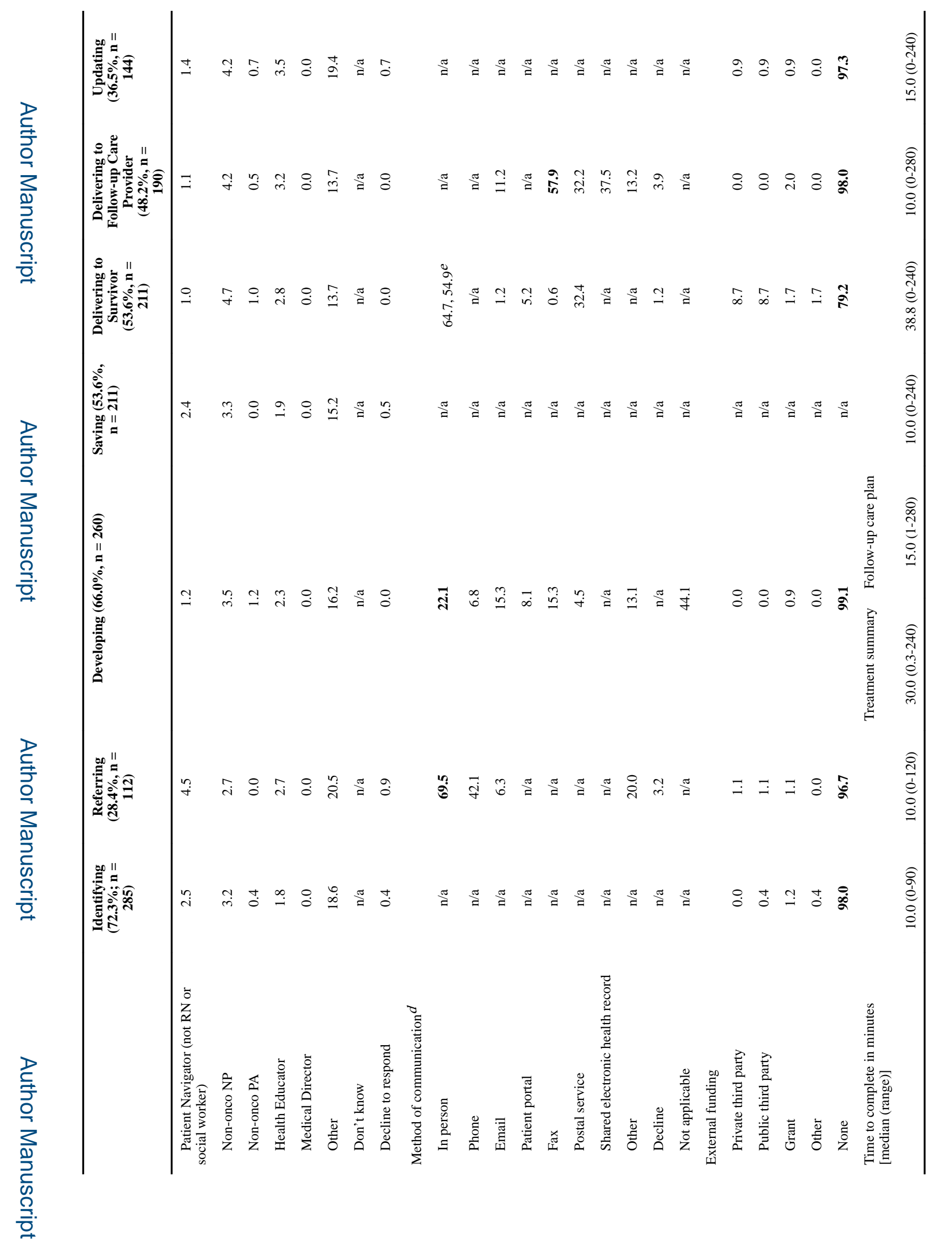

J Cancer Educ. Author manuscript; available in PMC 2020 June 01. 
Birken et al.

Page 16
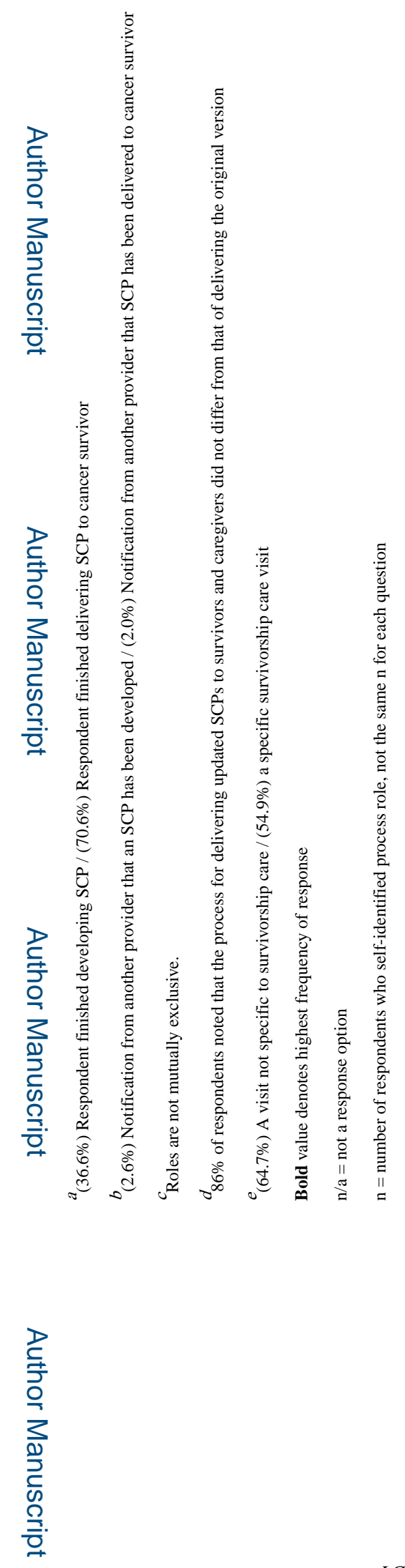

J Cancer Educ. Author manuscript; available in PMC 2020 June 01. 
Table 3

Characteristics of survivorship care plan (SCP) template creation and revision

\begin{tabular}{|c|c|}
\hline Characteristic & $\%$ \\
\hline \multicolumn{2}{|l|}{ Template used $(\mathrm{n}=229)$} \\
\hline Template developed within organization & 42.4 \\
\hline American Society of Clinical Oncology & 25.3 \\
\hline Journey Forward & 22.7 \\
\hline Other & 22.7 \\
\hline LIVESTRONG Care Plan & 4.8 \\
\hline Memorial Sloan Kettering Cancer Center & 0.4 \\
\hline Don’t know & 0.0 \\
\hline Decline to respond & 1.4 \\
\hline \multicolumn{2}{|l|}{ Resource for follow-up portion of SCP $(n=229)$} \\
\hline National Comprehensive Cancer Network guidelines & 80.3 \\
\hline American Society of Clinical Oncology guidelines & 60.3 \\
\hline American Cancer Society guidelines & 38.4 \\
\hline Institutional protocol & 24.5 \\
\hline Your own judgment & 11.4 \\
\hline Other & 8.3 \\
\hline Decline to respond & 0.4 \\
\hline \multicolumn{2}{|l|}{ How often are SCP templates revised? $(\mathrm{n}=228)$} \\
\hline Once a year & 38.6 \\
\hline Other & 25.4 \\
\hline Never & 17.5 \\
\hline Twice a year & 10.5 \\
\hline Four times a year & 5.3 \\
\hline Decline to respond & 2.6 \\
\hline \multicolumn{2}{|l|}{ How many people review changes to template? $(\mathrm{n}=182)$} \\
\hline More than one colleague & 63.2 \\
\hline One colleague & 20.3 \\
\hline No one & 15.9 \\
\hline Decline to respond & 1.6 \\
\hline \multicolumn{2}{|l|}{ Factors that prompt revision of SCP templates $(\mathrm{n}=182)$} \\
\hline Changes in guidelines or recommendations & 63.2 \\
\hline Changes in survivors', caregivers', or follow-up care providers' needs & 58.8 \\
\hline Other & 20.9 \\
\hline Decline to respond & 2.2 \\
\hline
\end{tabular}

J Cancer Educ. Author manuscript; available in PMC 2020 June 01. 